\title{
Wirksamkeit von Cannabidiol: Klinische Studien mit Cannabidiol und Cannabidiol-haltigen Extrakten
}

\author{
Samuel Büechi \\ Phytocon GmbH, Trogen, Schweiz

\section{Schlüsselwörter} \\ Cannabidiol · Cannabinoide $\cdot$ Klinische Studie
}

\section{Zusammenfassung}

Hintergrund: Tetrahydrocannabinol (THC) und Cannabidiol (CBD) sind die prominentesten Wirkstoffe aus der weiblichen Hanfpflanze (Cannabis sativa L.). Während sich die Kombination von THC- und CBD-reichen Extrakten dank ihrer analgetischen und antispastischen Wirkungen bei Patienten mit Multipler Sklerose etabliert hat, steht das Anwendungsprofil von CBD noch am Beginn seiner Erforschung. Methoden: Mittels PubMed-Recherchen und Studium der damit gefundenen Literatur wurden Publikationen von klinischen Studien gesucht, bei welchen Aspekte der Wirksamkeit von CBD oder CBD-reichen Extrakten als Monopräparate untersucht wurden. Es konnten 25 Studien identifiziert werden, welche die beschriebenen Kriterien erfüllen. Resultate und Schlussfolgerung: Die meisten ausgewählten Studien sind kontrollierte Pilotstudien mit 20 oder weniger Patienten pro Gruppe oder offene Studien. Evidenz für die Wirksamkeit von CBD gibt es bei Kindern mit therapieresistenter Epilepsie. Die Anzahl der Anfälle konnte bei Kindern mit Dravet-Syndrom in einer Dosierung von $20 \mathrm{mg}$ CBD/kg (zusätzlich zur Standardtherapie) signifikant reduziert werden. Evident ist auch die Wirksamkeit von Dosierungen zwischen 300 und 600 mg bei sozialen Angststörungen. Bezüglich der antipsychotischen Wirkung, der analgetischen Wirkung sowie der Wirkung auf den Schlaf sind die Resultate widersprüchlich.

๑c 2017 S. Karger GmbH, Freiburg

\section{Einleitung}

Tetrahydrocannabinol (THC) und Cannabidiol (CBD) sind die prominentesten Wirkstoffe aus den Blüten und Blättern der weiblichen Hanfpflanze. Die zur Gruppe der Cannabinoide gezählten Verbindungen liegen in der Pflanze überwiegend als Säure vor. Im Gegensatz zu THC hat CBD keine psychotrope Wirkung.

Während sich die Kombination von THC- und CBDreichen Extrakten wegen ihrer analgetischen und anti-

\author{
Keywords \\ Cannabidiol · Cannabinoids · Clinical Trial
}

\section{Summary}

Efficacy of Cannabidiol: Clinical Studies with Cannabidiol and Cannabidiol-Containing Extracts

Background: Tetrahydrocannabinol (THC) and cannabidiol (CBD) are the most prominent active ingredients of the female hemp plant (Cannabis sativa L.). While the combination of THC- and CBD-rich extracts has been established in patients with multiple sclerosis due to their analgesic and antispastic effects, the field of usage of CBD is still at the beginning of its exploration. Methods: By means of a PubMed search and study of the literature found, publications of clinical trials were identified in which aspects of the efficacy of preparations with CBD or CBD-rich extracts as single ingredients were investigated. 25 studies were identified which meet the criteria described. Results and Conclusion: Most selected trials are controlled pilot trials with 20 or fewer patients per group or open trials. There is high evidence for the efficacy of CBD in children with untreatable epilepsy. The number of seizures was significantly reduced in children with Dravet's syndrome at a dosage of $20 \mathrm{mg} \mathrm{CBD} / \mathrm{kg}$ (in addition to standard therapy). There is also evidence for the efficacy of dosages between 300 and $600 \mathrm{mg}$ for social anxiety disorders. With regard to the antipsychotic effect, the analgesic effect, and the effect on sleep, the results are contradictory.

spastischen Wirkungen bei Patienten mit Multipler Sklerose (MS) etabliert hat, steht das Anwendungsprofil von CBD noch am Beginn seiner Erforschung. Anerkannt und klinisch relevant ist bisher einzig die antiepileptische Wirkung - speziell bei Kindern mit Dravet-Syndrom.

Zurzeit (Stand: Juli 2017) laufen gemäss «Clinical Trials.gov», einem Service der US-amerikanischen National Institutes of Health, 76 klinische Studien, sodass sich der Erkenntnisstand zur klinischen Wirksamkeit von CBD in den nächsten Jahren noch mehrfach ändern wird.

\section{KARGER}

(c) 2017 S. Karger GmbH, Freiburs 


\section{Methoden}

Die Literatur wurde auf Basis einer Recherche in PubMed vom Juli 2017 zusammengestellt. Gesucht wurde mit dem Meshterm «Cannabidiol» und der Beschränkung auf klinische Studien. Diese Recherche wurde in den folgenden 2 Monaten noch mit anderen Suchstrategien erweitert. In den gefundenen Artikeln wurde nach weiteren relevanten Studien gesucht. Für die vorliegende Publikation wurden alle Arbeiten ausgewählt, in welchen Monopräparate mit CBD oder einem CBD-haltigen Extrakt in klinischen Studien auf ihre Wirksamkeit hin untersucht wurden.

\section{Resultate}

Mit der ersten PubMed-Recherche konnten 72 Arbeiten identifiziert werden. Viele davon betrafen klinische Studien mit Kombinationspräparaten wie Sativex ${ }^{\circledR}$, welche THC- und CBD-reiche Extrakte enthalten. Nach weiteren PubMed-Recherchen und bei Beschränkung auf klinische Studien, in welchen CBD oder CBD-haltige Extrakte als Monopräparat auf Aspekte der Wirksamkeit untersucht wurden, blieben 25 Publikationen übrig.

\section{Epilepsie}

In einer Doppelblindstudie wurden 120 Kinder mit Dravet-Syndrom mit 20 mg/kg Körpergewicht oder Placebo - in Kombination mit bestehender Medikation - behandelt. Während bei 43\% der Patienten unter CBD die Anzahl der Anfälle um mindestens 50\% reduziert wurde, waren es in der Placebo-Gruppe 27\%. Während 5\% der Patienten der Verum-Gruppe keinen Anfall hatten, waren es bei der Placebo-Gruppe 0\% [1].

Damit wurden die positiven Resultate von verschiedenen offenen Studien mit Patienten mit therapieresistenter Epilepsie bestätigt. In einer Studie wurden 214 Patienten zwischen 1 und 30 Jahren während 12 Wochen mit bis zu $50 \mathrm{mg}$ CBD/kg Körpergewicht in Kombination mit bestehender Medikation (Clobazam, Valproat, Stiripentol und andere) behandelt [2]. In einer retrospektiven Studie mit 74 Patienten zwischen 1 und 18 Jahren wurden diese mindestens 3 Monate (Durchschnitt: 6 Monate) mit CBD in einer Dosierung von 1-20 mg CBD/kg Körpergewicht in Form eines CBD-reichen Extraktes behandelt [3]. Eine positive Wirkung zeigte auch eine Studie, welche auf einer Umfrage bei Eltern von therapieresistenten epileptischen Kindern basiert, welche mit CBD behandelt wurden [4].

Bei einer älteren placebokontrollierten Doppelblindstudie mit 18 Patienten mit Huntington-Krankheit konnten mit einer durchschnittlichen Dosis von $700 \mathrm{mg}$ pro Tag während 6 Wochen weder Wirkungen noch unerwünschte Wirkungen nachgewiesen werden [5]. Eine teilweise Verbesserung konnte in einer placebokontrollierten Doppelblindstudie mit 15 Epilepsie-Patienten und 200 bzw. $300 \mathrm{mg}$ CBD täglich festgestellt werden [6]. Erste offene und erste kontrollierte Studien mit gesunden Probanden wurden in den 1970er-Jahren durchgeführt [7].

CBD wird auch bei einem seltenen Syndrom, welches Kinder mit Fieber und Infektion betrifft, eingesetzt - dem «Febrile Infection-Related Epilepsy Syndrome» (FIRES). Eine retrospektive Analyse von 7 Fällen weist auf eine mögliche Wirkung bei diesem Syndrom hin [8].

Weiter liegen bisher nicht publizierte kontrollierte Studien mit dem CBD-Produkt Epidiolex ${ }^{\circledR}$ vor. Eine Studie mit 120 Patienten mit Dravet-Syndrom soll eine signifikante Verminderung der Anfälle um 39\% mit 20 mg/kg Körpergewicht und um 13\% bei der Placebo-Gruppe gezeigt haben. Bei einer Studie mit 171 Patienten mit dem LennoxGastaut-Syndrom soll in derselben Dosierung eine Verminderung der Anfälle um 44\% beobachtet worden sein - gegenüber 22\% in der Placebo-Gruppe. In einer Dosisfindungsstudie mit 225 Patienten mit Lennox-GastautSyndrom wurden 10 und $20 \mathrm{mg}$ CBD pro kg und Placebo untersucht. $42 \%$ der Anfälle sollen mit $20 \mathrm{mg} / \mathrm{kg}$ verhindert worden sein, 37\% mit $10 \mathrm{mg} / \mathrm{kg}$ und 17\% mit Placebo [9].

\section{Anxiolytische Wirkung}

In einer Doppelblindstudie wurden 60 gesunde Probanden mit Placebo, Clonazepam (1 mg) und CBD (100, 300 und 900 mg) behandelt: Im Test «Öffentliches Reden in einer realen Situation» zeigte sich eine signifikante angstlösende Wirkung für Clonazepam für die Zeit der Rede (etwa 2,5 h nach Einnahme). In der Phase nach der Rede (3,5 h nach Einnahme) zeigten Clonazepam und 300 mg CBD eine angstlösende Wirkung - nicht aber $100 \mathrm{mg}$ und $900 \mathrm{mg}$ CBD [10].

24 Patienten mit sozialen Angststörungen wurden in einer Doppelblindstudie mit $600 \mathrm{mg}$ CBD oder Placebo behandelt: $\mathrm{CBD}$ zeigte eine signifikante vermindernde Wirkung bei der subjektiven Beurteilung der Angst und der kognitiven Beeinträchtigung [11].

Eine signifikante Verminderung der Angst konnte in einer placebokontrollierten Doppelblindstudie mit $10 \mathrm{~Pa}$ tienten mit sozialen Angststörungen und einer Verabreichung von $400 \mathrm{mg}$ CBD gezeigt werden [12]. Bei einer anderen kontrollierten Studie mit 40 Probanden und einer einmaligen Medikation mit $300 \mathrm{mg}$ CBD, $10 \mathrm{mg}$ Diazepam oder $5 \mathrm{mg}$ Ipsapiron reduzierten alle Substanzen die Angst im Zusammenhang mit einer simulierten öffentlichen Rede [13].

In einer placebokontrollierten Doppelblindstudie wurden 48 Probanden mit 32 mg CBD behandelt. Beim verwendeten Test werden Probanden mit Stromstössen so konditioniert, dass sie auf einen bestimmten Reiz (farbige Box) mit Angst reagieren. Danach lernen die Probanden, die Verbindung zwischen beiden Reizen «auszulöschen». Wenn CBD nach dieser Extinktionsphase gegeben wurde, verstärkte es im Vergleich zum Placebo die Dauerhaftig- 
keit der Auflösung der Verbindung zwischen den Reizen. Aus dem Resultat wurde auf einen möglichen therapeutischen Nutzen bei Angststörungen geschlossen [14].

Schizophrenie/psychotische Symptome bei Schizophrenie

Das Pharmaunternehmen GW Pharmaceuticals berichtete 2015 über den Einsatz von CBD bei schizophrenen Psychosen. 88 Patienten erhielten in dieser Studie zusätzlich zur bisherigen antipsychotischen Medikation CBD oder Placebo. Dreimal mehr Patienten der CBDGruppe sollen auf die Therapie angesprochen haben. Die Studie wurde noch nicht publiziert [15].

In einer Doppelblindstudie wurden Amisulprid und CBD (je bis $800 \mathrm{mg}$ täglich) über einen Zeitraum von 28 Tagen bei 39 Patienten mit psychotischen Symptomen bei Schizophrenie untersucht. Unter beiden Substanzen konnte eine signifikante Verminderung der Symptome beobachtet werden. Unter $\mathrm{CBD}$ wurde zudem eine signifikante $\mathrm{Zu}$ nahme des Serum-Anandamid-Gehaltes beobachtet [16].

In einer kontrollierten Studie mit 28 SchizophreniePatienten konnte bei einmaliger Gabe von 300 und $600 \mathrm{mg}$ CBD mit dem «Farbe-Wort-Interferenz-Test nach Stroop» im Vergleich zu «keiner Therapie» keine Wirkung festgestellt werden [17].

Bei einer offenen Pilotstudie mit 6 Patienten mit Parkinson und Psychosen zeigte sich bei einer Dosierung von 150 mg CBD pro Tag eine Abnahme der psychotischen Symptome [18].

Eine offene Studie mit 3 Schizophrenie-Patienten und Gabe von 40-1280 mg CBD über 55 Tage zeigte bei einem Patienten eine Verbesserung [19].

\section{Schlaf}

In einer Doppelblindstudie mit 15 Patienten, welche unter Schlaflosigkeit litten und 40, 80 und 160 mg CBD bzw. Placebo einnahmen, zeigte sich eine signifikante Verlängerung der Schlafdauer mit $160 \mathrm{mg}$. 40 und $80 \mathrm{mg}$ sowie das Placebo zeigten keine entsprechende Wirkung [7].

In einer Doppelblindstudie wurden 24 Patienten (hiervon 18 mit der Diagnose MS) mit THC, CBD, der 1:1-Mischung derselben und Placebo behandelt. Die individuellen Dosierungen lagen zwischen 2,5 und $120 \mathrm{mg}$ pro Tag. Die Resultate für CBD waren unauffällig bezüglich dem Parameter Schlafqualität [20].

In einer Crossover-Studie mit 34 Patienten mit chronischen Schmerzen wurden THC, CBD, die 1:1-Mischung derselben und Placebo untersucht. Die Dosierung war individuell und reichte von 1-33 Sprühstössen (zu 2,5 mg THC, 2,5 mg CBD oder 2,5 mg THC und 2,5 mg CBD) pro Tag. THC, CBD und die Kombination derselben zeigten eine signifikante Wirkung betreffend der Schlafqualität. Die Zahl der Patienten, welche gut geschlafen haben, betrug 55\% für
THC und CBD ( $p<0,001)$, 43\% für THC ( $<<0,001$ ), 37\% für CBD $(\mathrm{p}<0,05)$ und $17 \%$ für Placebo. Die Dosierungen von CBD lagen zwischen 1 und 31 Sprühstössen (3-78 mg); der Median lag bei etwa 8 Sprühstössen (20 mg) [21].

\section{Schmerzen}

In einer Crossover-Studie mit 34 Patienten mit chronischen Schmerzen wurden THC, CBD, die 1:1-Mischung derselben sowie Placebo untersucht: THC und die Kombination von THC und CBD zeigten eine signifikante Wirkung verglichen mit Placebo - CBD hingegen nicht. Die Dosierungen waren individuell und reichten von 1-33 Sprühstössen (zu je 2,5 mg THC oder CBD oder zu 2,5 $\mathrm{mg}$ THC und 2,5 $\mathrm{mg}$ CBD) pro Tag [21].

Eine Doppelblindstudie untersuchte 24 Patienten mit nicht behandelbaren neurogenen Schmerzen (18 davon mit MS). Als Medikation dienten THC, CBD, eine 1:1-Mischung derselben sowie ein Placebo. Die Dosierungen lagen zwischen 2,5 und $120 \mathrm{mg}$ pro Tag. Bei Schmerzen konnte sowohl für THC als auch für CBD eine signifikante Wirkung festgestellt werden, welche beim CBD aber nur auf den Resultaten von 12 Patienten basiert. Die Resultate für $\mathrm{CBD}$ waren unauffällig für andere Parameter wie Krämpfe oder Schlafqualität [20].

\section{Tabaksucht}

In einer Doppelblindstudie mit 24 Rauchern wurde CBD gegenüber Placebo untersucht. Die Prüfmedikamente konnten ad libitum inhaliert werden. Innerhalb einer Woche konnten die gerauchten Zigaretten bei CBDGabe - nicht aber mit dem Placebo - signifikant um etwa $40 \%$ vermindert werden [22].

\section{Transplantationen}

In einer offenen Studie wurden 50 Transplantationspatienten 37 Tage lang mit $300 \mathrm{mg}$ CBD pro Tag behandelt. Im retrospektiven Vergleich zeigte sich eine Verminderung der Abstossungsreaktionen [23].

\section{Parkinson}

In einer Doppelblindstudie wurden 21 Patienten mit Bewegungsstörungen mit CBD (75 oder $300 \mathrm{mg}$ ) oder Placebo behandelt. Mit dem «Parkinson's Disease Questionnaire» wurden keine signifikanten Effekte auf die Krankheit beobachtet. Hingegen konnte die Lebensqualität durch $300 \mathrm{mg}$ CBD pro Tag im Vergleich zu Placebo signifikant verbessert werden. $75 \mathrm{mg}$ CBD pro Tag zeigten keine solche Wirkung [24].

In einer offenen Pilotstudie mit 5 Patienten, welche unter dystonischen Bewegungsstörungen litten, wurde CBD 
in Dosierungen zwischen 100 und 600 mg pro Tag während 6 Wochen - zusätzlich zur bestehenden Medikation gegeben. Die Dystonie wurde bei allen Patienten um 20-50\% vermindert. Die Symptome Hypokinese und Tremor verschlechterten sich bei 2 Patienten. Daraus wurde der Schluss gezogen, dass CBD antidystonisch wirkt, aber die Symptome Tremor und Hypokinese verstärkt [25].

\section{Augeninnendruck}

Eine Crossover-Studie mit 6 Patienten mit erhöhtem Augeninnendruck und der Gabe von $5 \mathrm{mg}$ THC, 20 und $40 \mathrm{mg}$ CBD sowie Placebo zeigte, dass nur die $5 \mathrm{mg}$ THC den Augeninndruck vermindern konnten, während $20 \mathrm{mg}$ CBD keine Wirkung zeigten und $40 \mathrm{mg}$ CBD den Druck erhöhten [26].

\section{Verträglichkeit}

Bei Dosierungen bis $20 \mathrm{mg}$ kommen unerwünschte Wirkungen gelegentlich vor. Bei Dosierungen von $20 \mathrm{mg} / \mathrm{kg}$ Körpergewicht werden sie sehr häufig beobachtet. Auf der Basis der neuesten und grössten kontrollierten CBD-Studie kann für diese hohe Dosierung Folgendes festgestellt werden: Unerwünschte Ereignisse wurden in 93\% der CBDGruppe und 75\% der Placebo-Gruppe beobachtet. Bei 75\% der unerwünschten Ereignisse der CBD-Gruppe standen die Ereignisse wahrscheinlich im Zusammenhang mit der Studie - bei der Placebo-Gruppe waren dies nur 36\%. Häufige unerwünschte Wirkungen (mit $>10 \%$ Frequenz) in der CBD-Gruppe waren: Erbrechen, Müdigkeit, Fieber, Infektionen der oberen Luftwege, verminderter Appetit, Krämpfe, Lethargie, Schläfrigkeit und Durchfall. In der CBD-Gruppe beendeten 8 Patienten die Studie wegen unerwünschten Ereignissen; in der Placebo-Gruppe war es 1 Patient. Aufgrund transienter erhöhter Aminotransferase-Spiegel kann ein metabolischer Stress auf die Leber vermutet werden [1].

\section{Diskussion}

Am besten ist die Wirkung von CBD beim Dravet-Syndrom dokumentiert. Zusammen mit der bestehenden Standardtherapie kann CBD in einer Dosierung von 20 $\mathrm{mg} / \mathrm{kg}$ Körpergewicht die Anfallshäufigkeit signifikant senken [1]. Weitere noch nicht publizierte kontrollierte Studien der Firma GW Pharmaceuticals sollen auch die Wirkung beim Lennox-Gastaut-Syndrom bestätigen [9].

In einer offenen Studie wurde die Verträglichkeit von CBD in Dosierungen von bis zu $50 \mathrm{mg} / \mathrm{kg}$ Körpergewicht in Kombination mit der bestehenden Medikation bei 214 Kindern mit unbehandelbarer Epilepsie untersucht. Erwähnenswert sind auch eine retrospektive Studie mit 74 Epilepsie-Patienten im Alter von 1-18 Jahren [3] und eine
Umfrage bei Eltern von 117 Kindern mit therapieresistenter Epilepsie [4]. Auch die Resultate dieser drei offenen Studien weisen auf eine Wirksamkeit bei speziellen Epilepsieformen und eine angemessene Verträglichkeit hin. Bei Patienten mit Epilepsie, welche mit herkömmlichen Antiepileptika nicht erfolgreich therapiert werden können, ist ein Versuch mit CBD durchaus empfehlenswert. Beachtet werden muss dabei aber auch der finanzielle Aspekt. CBD ist nicht kassenpflichtig, und nicht alle Patienten können sich diesen Versuch leisten.

Zwei kleinere Studien zur Wirksamkeit von CBD bei sozialen Angststörungen zeigen, dass 600 und $400 \mathrm{mg}$ CBD die Angst vermindern [11, 12]. In einer grösseren Studie konnte dies für $300 \mathrm{mg}$ - nicht aber für 100 und 900 mg - bestätigt werden [10]. Die klinische Relevanz dieser Erkenntnis ist noch offen. Bisher wurde nur die Wirkung auf «öffentliches Sprechen» untersucht.

Bezüglich einer möglichen antipsychotischen Wirkung kann festgestellt werden, dass CBD in einer Dosierung von $800 \mathrm{mg}$ eine ähnliche Wirkung hat wie Amisulprid. Für beide Substanzen konnte bei 39 Patienten eine signifikante Verminderung der psychotischen Symptome bei Schizophrenie beobachtet werden. Die unter CBD beobachtete signifikante Zunahme des Serum-AnandamidGehaltes könnte ein Hinweis auf einen möglichen Wirkmechanismus sein [16]. Keine signifikante Wirkung wurde bei einmaliger Gabe von 300 oder $600 \mathrm{mg}$ CBD bei 28 Schizophrenie-Patienten beobachtet [17]. Eine noch nicht publizierte Studie mit 88 Patienten von GW Pharmaceuticals zeigt dagegen wiederum eine antipsychotische Wirkung [15]. In einem systematischen Review wurde festgestellt, dass die Evidenz für eine Wirkung auf die kognitive Funktion bei Schizophrenie-Patienten beschränkt ist [27]. Dasselbe gilt für die antipsychotische Wirkung.

Die Resultate zur Wirkung von CBD auf den Schlaf sind widersprüchlich. In einer placebokontrollierten Doppelblindstudie mit 15 Patienten, welche unter Schlaflosigkeit litten, zeigte sich nur mit $160 \mathrm{mg}$ CBD eine signifikante Verlängerung der Schlafdauer - nicht aber mit 40 und $80 \mathrm{mg}$. Alle drei CBD-Dosierungen verminderten die Erinnerung an Träume signifikant. Die Relevanz dieser Beobachtung auf den Schlaf ist allerdings unklar. Die Wirkung könnte auf einer Verminderung der Kapazität zu träumen oder einer Verminderung der Erinnerung an die Träume basieren - z.B. über eine Verminderung der Aufwachphasen [7]. In einer anderen placebokontrollierten Doppelblindstudie mit 24 Patienten zeigte sich bei individueller Dosierung keine Wirkung von CBD auf die Schlafqualität [20]. In einer Crossover-Studie mit $34 \mathrm{~Pa}$ tienten zeigte CBD dagegen eine positive Wirkung auf die Schlafqualität [21]. Es ist denkbar, dass die unterschiedlichen Resultate bedingt sind durch individuell unterschiedliche Reaktionen auf CBD; d.h., dass CBD bei gewissen Patienten den Schlaf verbessert oder verlängert hat 
und bei anderen nicht. Mit Hinblick auf die Studie von Carlini und Cunha [7] könnte aber auch vermutet werden, dass nur höhere Dosierungen eine objektivierbare schlaffördernde Wirkung haben.

Ähnlich ist die Situation bei Schmerzen. Eine placebokontrollierte Doppelblindstudie zeigt, dass CBD - im Gegensatz zu THC - bei chronischen Schmerzen und individueller Dosierung keine analgetische Wirkung hat [21]. In einer anderen placebokontrollierten Doppelblindstudie mit vorwiegend MS-Patienten konnte sowohl für THC als auch für CBD eine signifikante Wirkung festgestellt werden [20]. Bei CBD basiert dieses Resultat allerdings nur auf den Resultaten von 12 Patienten. Vielleicht hat CBD nur bei einzelnen Patienten mit bestimmten chronischen Schmerzen eine analgetische Wirkung. Patienten mit MS könnten dabei mehr profitieren als solche mit anderen Schmerzformen. Es gibt keine klinischen Hinweise, dass CBD auch bei akuten Schmerzen oder chronischen Schmerzen der Gelenke und Muskeln helfen könnte.

$\mathrm{Zu}$ der vielfach ausgelobten entzündlichen Wirkung und der Wirkung von CBD auf Tumore liegen keine kli- nischen Untersuchungen vor. Bei der Wirkung auf Tumore gibt es allerdings eine beachtliche Anzahl positiver präklinischer Daten [28].

Falls die Anwendung von CBD bei Patienten mit Schmerzen, Schlafstörungen oder anderen Krankheiten in Betracht gezogen wird, empfiehlt sich folgendes Vorgehen: Die Dosierung sollte langsam gesteigert und individuell eingestellt werden - z.B. basierend auf der Beurteilung der Verträglichkeit und Wirksamkeit durch den Patienten. Die Tagesdosis kann aufgeteilt werden in zwei Dosen (morgens und abends). CBD hat Wechselwirkungen mit anderen Medikamenten. Eine Verstärkung der beruhigenden Wirkung anderer Medikamente muss erwartet werden. Vorsicht ist geboten, wenn zusätzlich Arzneimittel mit potenziell lebertoxischer Wirkung eingenommen werden.

\section{Disclosure Statement}

Der Autor war für die Firmen AI LAB Swiss AG und Swiss Cannabis SA tätig, welche in der Produktion und dem Handel von CBD aktiv sind.

\section{Literatur}

1 Devinsky O, Cross JH, Laux L, et al: Trial of cannabidiol for drug-resistant seizures in the Dravet syndrome. N Engl J Med 2017;376: 2011-2020.

2 Devinsky O, Marsh E, Friedman D, et al: Cannabidiol in patients with treatment-resistant epilepsy: an open-label interventional trial. Lancet Neurol 2016;15:270-278.

3 Tzadok M, Uliel-Siboni S, Linder I, et al: CBD-enriched medical cannabis for intractable pediatric epilepsy: the current Israeli experience. Seizure 2016;35:41-44.

4 Hussain SA, Zhou R, Jacobson C, et al: Perceived efficacy of cannabidiol-enriched cannabis extracts for treatment of pediatric epilepsy: a potential role for infantile spasms and Lennox-Gastaut syndrome. Epilepsy Behav 2015;47:138-141.

5 Consroe P, Laguna J, Allender J, et al: Controlled clinical trial of cannabidiol in Huntington's disease. Pharmacol Biochem Behav 1991;40:701-708.

6 Cunha JM, Carlini EA, Pereira AE, et al: Chronic administration of cannabidiol to healthy volunteers and epileptic patients. Pharmacology 1980;21:175-185.

7 Carlini EA, Cunha JM: Hypnotic and antiepileptic effects of cannabidiol. J Clin Pharmacol 1981;21(suppl):417S-427S.

8 Gofshteyn JS, Wilfong A, Devinsky O, et al: Cannabidiol as a potential treatment for febrile infection-related epilepsy syndrome (FIRES) in the acute and chronic phases. J Child Neurol 2017;32:35-40.

9 O'Connell BK, Gloss D, Devinsky O: Cannabinoids in treatment-resistant epilepsy: a review. Epilepsy Behav 2017;70:341-348.

10 Zuardi AW, Rodrigues NP, Silva AL, et al: Inverted U-shaped dose-response curve of the anxiolytic effect of cannabidiol during public speaking in real life. Front Pharmacol 2017;8: 259.
11 Bergamaschi MM, Queiroz RH, Chagas MH, et al: Cannabidiol reduces the anxiety induced by simulated public speaking in treatment-naïe social phobia patients. Neuropsychopharmacology 2011;36:1219-1226.

12 Crippa JA, Derenusson GN, Ferrari TB, et al: Neural basis of anxiolytic effects of cannabidiol (CBD) in generalized social anxiety disorder: a preliminary report. J Psychopharmacol 2011;25:121-130.

13 Zuardi AW, Cosme RA, Graeff FG, Guimarães FS: Effects of ipsapirone and cannabidiol on human experimental anxiety. J Psychopharmacol 1993;7(suppl):82-88.

14 Das RK, Kamboj SK, Ramadas M, et al: Cannabidiol enhances consolidation of explicit fear extinction in humans. Psychopharmacology 2013;226:781-792.

15 GW Pharmaceuticals: GW Pharmaceuticals announces positive proof of concept data in schizophrenia. 15.09.2015. https://www. gwpharm.com/about-us/news/gw-pharmaceuticals-announces-positive-proof-concept-dataschizophrenia.

16 Leweke FM, Piomelli D, Pahlisch F, et al: Cannabidiol enhances anandamide signaling and alleviates psychotic symptoms of schizophrenia. Transl Psychiatry 2012;2:e94.

17 Hallak JE, Machado-de-Sousa JP, Crippa JA, et al: Performance of schizophrenic patients in the Stroop Color Word Test and electrodermal responsiveness after acute administration of cannabidiol (CBD). Rev Bras Psiquiatr 2010;32:56-61.

18 Zuardi AW, Crippa JA, Hallak JE, et al: Cannabidiol for the treatment of psychosis in Parkinson's disease. J Psychopharmacol 2009; 23:979-983.

19 Zuardi AW, Hallak JE, Dursun SM, et al: Cannabidiol monotherapy for treatment-resistant schizophrenia. J Psychopharmacol 2006;20:683-686.
20 Wade DT, Robson P, House H, et al: A preliminary controlled study to determine whether whole-plant cannabis extracts can improve intractable neurogenic symptoms. J. Clin Rehabil 2003;17:21-29.

21 Notcutt W, Price M, Miller R, et al: Initial experiences with medicinal extracts of cannabis for chronic pain: results from 34 ' $\mathrm{N}$ of 1' studies. Anaesthesia 2004;59:440-452.

22 Morgan CJ, Das RK, Joye A, et al: Cannabidiol reduces cigarette consumption in tobacco smokers: preliminary findings. Addict Behav 2013;38:2433-2436.

23 Yeshurun M, Shpilberg O, Herscovici C, et al: Cannabidiol for the prevention of graft-versus-host-disease after allogeneic hematopoietic cell transplantation: results of a phase II study. Biol Blood Marrow Transplant 2015; 21:1770-1775.

24 Chagas MH, Zuardi AW, Tumas V, et al: Effects of cannabidiol in the treatment of patients with Parkinson's disease: an exploratory double-blind trial. J Psychopharmacol 2014;28: 1088-1098.

25 Consroe P, Sandyk R, Snider SR: Open label evaluation of cannabidiol in dystonic movement disorders. Int J Neurosci 1986;30:277282.

26 Tomida I, Azuara-Blanco A, House H, et al: Effect of sublingual application of cannabinoids on intraocular pressure: a pilot study. J Glaucoma 2006;15:349-353.

27 Osborne AL, Solowij N, Weston-Green K: A systematic review of the effect of cannabidiol on cognitive function: relevance to schizophrenia. Neurosci Biobehav Rev 2017;72:310 324

28 Pisanti S, Malfitano AM, Ciaglia E, et al: Cannabidiol: state of the art and new challenges for therapeutic applications. Pharmacol Ther 2017;175:133-150. 